\title{
Safety and adherence of Umezu polyphenols in the Japanese plum (Prunus mume) in a 12-week double-blind randomized placebo-controlled pilot trial to evaluate antihypertensive effects
}

\author{
Shigeki Takemura · Kouichi Yoshimasu • Jin Fukumoto • Kanae Mure • \\ Nobuhiro Nishio • Kunihiro Kishida - Fumiko Yano • Takahiko Mitani • \\ Tatsuya Takeshita $\cdot$ Kazuhisa Miyashita
}

Received: 24 April 2014/Accepted: 11 August 2014/Published online: 13 September 2014

(C) The Japanese Society for Hygiene 2014

\begin{abstract}
Objectives Medications or lifestyle changes to prevent or improve hypertension often press considerable efforts on patients suffering from mild hypertension. Capsules including Umezu polyphenols (UP), polyphenols in Japanese plums, may help them to control their blood pressure (BP). The aim of this study is to evaluate the effectiveness of UP on BP and its safety.

Methods A total of 15 healthy workers without antihypertensive medication who had some concerns about their $\mathrm{BP}$, preferably normal-high BP or hypertension level 1, were randomized in a double-blind manner into UP ingesting and placebo groups. Each subject was instructed to take four capsules daily for 12 weeks (daily UP dose, $800 \mathrm{mg}$ for the UP ingesting group; and $0 \mathrm{mg}$ for the placebo group). These subjects were followed for 12 weeks, and their BP both at home and at the examination site, as well as self-perceived quality-of-life outcomes
\end{abstract}

A part of this study was presented in the 72nd Annual Meeting of the Japanese Society of Public Health on October 23-25, 2013.

S. Takemura $(\bowtie) \cdot$ K. Yoshimasu · J. Fukumoto $\cdot$ K. Miyashita Department of Hygiene, School of Medicine, Wakayama Medical University, 811-1 Kimiidera, Wakayama 641-8509, Japan

e-mail: steakmmi@hotmail.com

K. Mure $\cdot$ N. Nishio $\cdot$ T. Takeshita

Department of Public Health, School of Medicine, Wakayama

Medical University, Wakayama, Japan

K. Kishida - F. Yano - T. Mitani

Biology-Oriented Science and Technology, Kinki University,

Wakayama, Japan

T. Mitani

Wakayama Industry Promotion Foundation, Wakayama, Japan and possible side effects, was monitored during that period. Group $\times$ time interactions on BP changes were examined. Results All of the 15 subjects completed the 12-week intervention trial. The BP changes did not significantly differ between the UP ingesting and placebo groups, neither at the examination site nor at home. But during the study period, no adverse effects were observed.

Conclusions No remarkable effect of UP on BP was observed. However, a higher dose of UP was confirmed safe and high in adherence in this 12-week randomized controlled trial. Its effect on BP and other outcomes shall be confirmed in a larger sample.

Keywords Hypertension - Prevention - Japanese plum . Polyphenol · Randomized placebo-controlled trial

\section{Introduction}

Hypertension is one of the major risk factors associated with cardio- and cerebrovascular diseases, and has a strong impact on global health. In Japan, the mortality rates from those diseases have declined since the second half of 1960s, which is considered to be partially due to the decline in blood pressure (BP) levels and the lower prevalence of hypertension during the years 1965-1990 [1]. However, it is estimated that there are still 40 million people with hypertension in Japan [2]. It is a serious concern that many of those with hypertension do not receive appropriate treatments for hypertension, especially among young and middle-aged people. When hypertension is defined as $140 \mathrm{mmHg}$ or more of systolic blood pressure (SBP) and/or $90 \mathrm{mmHg}$ or more of diastolic blood pressure (DBP), about 80-90\% of Japanese in their thirties and forties with hypertension are considered not to have 
received any treatment [2]. Since most of the existing antihypertensive drugs often show some adverse effects, their applications are limited, especially for those with mild hypertension who are approaching clinically dangerous levels. Therefore, those people should lower their BPs, at least by changing their lifestyles to healthier ones. Although low-salt diets and exercise are proven to be effective against hypertension, following through with them is often burdensome for those people. Regarding dietary factors, high consumption of fruits and vegetables has been correlated with a decrease in cardiovascular disease [3-5]. However, intake of fruits and vegetables cannot be strongly recommended for patients with serious renal failure, since such diets may induce hyperkalemia in them [2]. Furthermore, excessive intake of fruits with high concentrations of glucose should be avoided by people with diabetes mellitus.

On the other hand, significant protective effects of antioxidants included in vegetables and fruits have been noted [6], while interventional trials of antioxidants have provided mixed results, some showing deleterious ones [6, 7]. Among those antioxidants, polyphenols from tea, wine, grapes, berries and other plants have been shown to activate endothelial cells and to increase the formation of potent vasoprotective factors, including nitric oxide (NO) and endothelium-derived hyperpolarizing factors. In addition, polyphenols interfere with mechanisms that lead to inflammation, platelet aggregation, and endothelial apoptosis, and contribute to the prevention of endothelial dysfunction, which is known to play a central role in the pathogenesis of cardiovascular diseases [8-11].

Polyphenols are found abundantly in fruits such as plums or prunes. Interestingly, the prune was reported to have protective effects against cardiovascular diseases, inducing significant reductions of BP and reducing serum cholesterol and LDL [12]. This effect was considered to be due to the antioxidant constituents of prunes [12]. If such constituents were added to common soft drinks, people with mild hypertension might successfully reduce their BP without having to make considerable efforts. In this regard, Japanese plums, especially the well-known products of Wakayama Prefecture in Japan, have been reported to be effective for improving human health including cardiovascular conditions $[13,14]$.

Based on the above-mentioned background, we performed a double-blind randomized study in 2011, using Umezu polyphenols (UP), i.e., polyphenols extracted from Japanese plums (Japanese name, Ume; botanical name, Prunus mume), which unsuccessfully revealed BP-lowering effects [15]. This may be due to some factors including a relatively short study period (5 weeks) and insufficiency of the dose (200 mg of UP daily). Then, we conducted a 12-week RCT, with a higher dose of UP.

\section{Materials and methods}

Subjects

We conducted a preliminary study using a sample of workers (clerical workers, university faculty members, physicians and comedicals) in our principal study center from December 2012 to March 2013. We recruited study participants interested in prevention or control of their hypertension, especially from those with normal-high BP (SBP, 130-139 $\mathrm{mmHg}$; or DBP, $85-89 \mathrm{mmHg}$ ) or hypertension grade 1 (SBP, $140-159 \mathrm{mmHg}$; or DBP, $90-99 \mathrm{mmHg}$ ) taking no antihypertensive agents [2]. Those who met the following exclusive criteria could not participate in the study: (1) those who ate 2 or more Umeboshi (pickled plums) per day, (2) those under medication for hypertension, (3) those with serious somatic disorders including cerebrovascular disease, ischemic heart disease, cancer, and diabetes mellitus, (4) those who were pregnant or within 1 year after delivery, (5) those with ingestion difficulty, (6) those having night-shift work with night duty, and (7) those who could not participate in the periodical physical measurement.

A total of 15 workers [ 13 men and 2 women; mean (SD) of age, 43.2 (12.4) years] participated in the study. They were randomly divided into 2 groups [Group A $(n=8)$ and Group B $(n=7)]$ whose sex and age distributions were adjusted to be as equal as possible (Fig. 1). Group A received placebo capsules ( $0 \mathrm{mg}$ of UP per capsule), while Group B received capsules containing UP (200 mg of UP

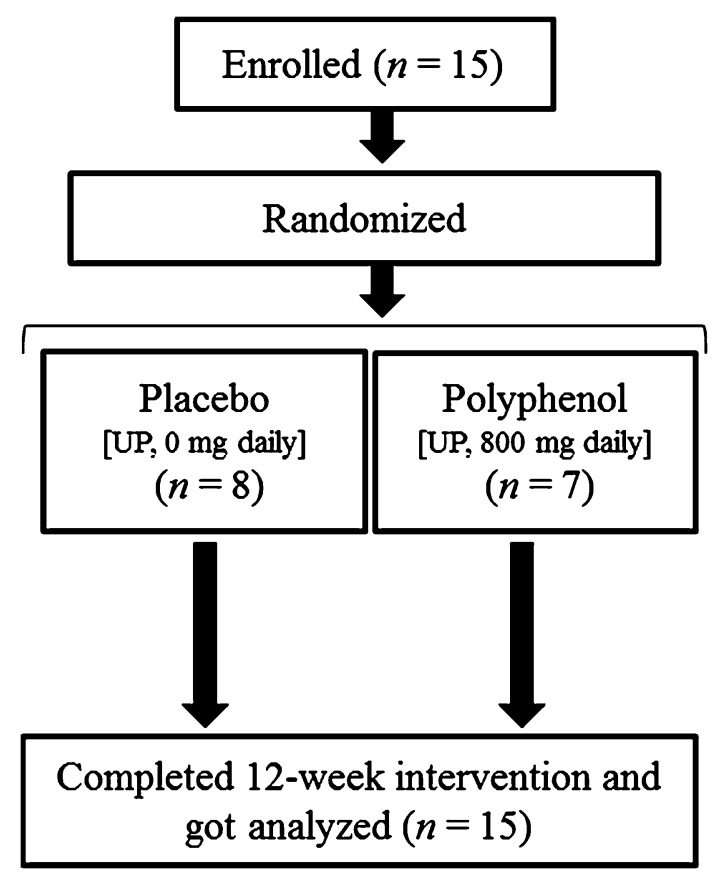

Fig. 1 Flowchart of subject enrollment, intervention and analysis. UP, Umezu polyphenols 
per capsule). They were instructed to take four capsules daily for 12 weeks, and the total daily dosage of UP was 0 and $800 \mathrm{mg}$ in Group A and Group B, respectively. These dosages were determined based on the study results of rats conducted in advance (data not shown). The study period of 12 weeks was determined, pursuant to the principle the Consumer Affairs Agency, Government of Japan (formerly the Ministry of Health, Labor and Welfare, Government of Japan) shows. The Agency generally requires an intervention trial of 12 weeks or longer to approve Tokuho, or foods for specified health uses (e.g., BP lowering) [16].

The mean (SD) of age among the 15 participants was 43.2 (12.4) years. When the study began, at the examination site, 1 of the 15 was at hypertension grade 2 (SBP, 160-179 mmHg, or DBP, 100-109 mmHg) (who was at hypertension 1 in subsequent measurements), 6 at hypertension grade 1, and 3 at normal-high BP at the examination site; at home, 6 were at hypertension grade 1 and 3 at normal-high BP.

Neither the examiners nor subjects knew which kind of capsules were being taken (i.e., double-blind design) throughout the study period. The current study was approved by the Institutional Review Board of Wakayama Medical University. Informed consent was acquired from all the participants in written form.

\section{Extraction of polyphenols}

Fruit samples of Prunus mume cv. 'Nanko' were randomly collected from one fixed tree grown at the experimental orchard of the Laboratory of Japanese Plum, Fruit Tree Experiment Station, Wakayama Research Center of Agriculture, Minabe Town, from 2006 to 2008, and stored in polyethylene bags at $-20{ }^{\circ} \mathrm{C}$ until analysis.

Since details surrounding the determination of total polyphenols and the preparation of polyphenol fractions through a biochemical experimental system are beyond the current study's scope, they will be described elsewhere [17, 18]. In brief, the Folin-Ciocalteu method with gallic acid as a standard was used for the determination of total polyphenols, and a batch method was adopted for the preparation of polyphenol fractions.

Umezu polyphenols were found to show many chromatographically isolated peaks. Our experimental analysis clarified that the UP were chemically composed of hydroxycinnamic acid derivatives. Four aglycones were identified as $c i s$ - $p$-coumaric acid, trans-p-coumaric acid, caffeic acid, and ferulic acid. Those aglycones are bound to various kinds of organic acids or sugars, and exist as the ingredients of UP. The extracted UP were sent to a supplement manufacturer (Kyoto Nourishment Natural Chemical Laboratory Co., Ltd., Kyoto, Japan), and added into supplement capsules.
Blood pressure, and physical and mental conditions

Study subjects were asked to measure their SBP and DBP early in the morning (after urinating and/or defecating, and before eating breakfast) and before sleep at night every day (it was not restricted for subjects to consume alcoholic beverages or take a bath before measuring the night BP measurement); SBP and DBP of the right arm were measured with an automatic sphygmomanometer (HEM-747IC, Omron, Tokyo, Japan), twice in a sitting position with arms supported at the right atrium level. When the difference of SBP between the first measure and the second measure was $10 \mathrm{mmHg}$ or greater, BP was measured one more time (a total of three times). At the beginning of the study, the principal investigator (ST) individually instructed each study subject how to measure BP and record it in the paper-based journal at home.

Blood pressure was measured at the examination site in the morning (8:30 a.m. to 0:00 p.m.) at baseline and 1, 2, 4, $6,8,10$ and 12 weeks later. At the examination site, SBP and DBP were measured with an automatic sphygmomanometer (HEM-907, Omron, Tokyo, Japan) by research staff (a trained physician or nurse), twice in a sitting position with arms supported at the right atrium level. When the difference of SBP between the first measure and the second measure was $10 \mathrm{mmHg}$ or greater, BP was measured one more time (a total of three times).

To evaluate changes in subjective health conditions, subjects' perceived physical and mental quality of life (QOL) was assessed at baseline and 4, 8 and 12 weeks later by SF-8 [19]. A higher SF-8 score [physical component summary (PCS) or mental component summary (MCS)] indicates a better physical/mental condition. In Japanese people, the average score and the standard deviation are 50 points and 10 points, respectively.

To ensure the safety of subjects, they were asked about their conditions including signs and symptoms at each of their visits of the examination site. When they reported some signs and symptoms, they were further asked if these signs and symptoms manifested themselves after taking UP or placebo capsules.

\section{Statistical analysis}

We calculated the mean value of subject-measured BP for each day, including all measured values, then we defined the $\mathrm{BP}$ at the beginning of the study (week 0 ) as the mean value of the BP measured on the first day of the measurement, and the BP at each week (weeks 1-12) as the mean value of all 7 days of the week, excluding the data for week 0 . We also calculated the mean value of staff-measured BP, for each follow-up session (weeks 1, 2, 4, 6, 8, 10 and 12, if available) as well as the beginning of the study period (week 0 ). These BP values were chosen as outcome variables. As for SF-8, PCS and MCS were outcome variables. 
Table 1 Characteristics of study subjects at baseline

\begin{tabular}{|c|c|c|c|}
\hline & $\begin{array}{l}\text { Placebo } \\
\text { group } \\
(n=8)\end{array}$ & $\begin{array}{l}\text { Polyphenol } \\
\text { group } \\
(n=7)\end{array}$ & $p^{a}$ \\
\hline $\begin{array}{c}\text { Daily dose of Umezu } \\
\text { polyphenols (mg) }\end{array}$ & 0 & 800 & - \\
\hline Age (years) & $42.3(12.2)$ & $44.3(13.5)$ & 0.764 \\
\hline Sex: male $[n(\%)]$ & $7(87.5)$ & $6(85.7)$ & 1.000 \\
\hline Height $(\mathrm{cm})$ & $169.7(6.6)$ & $166.7(8.0)$ & 0.437 \\
\hline Weight (kg) & $71.4(17.0)$ & $69.6(14.9)$ & 0.835 \\
\hline Body mass index $\left(\mathrm{kg} / \mathrm{m}^{2}\right)$ & $24.5(4.2)$ & $24.8(3.4)$ & 0.880 \\
\hline Waist circumference $(\mathrm{cm})$ & $89.0(10.9)$ & $86.4(13.2)$ & 0.688 \\
\hline Hip circumference $(\mathrm{cm})$ & $100.9(9.0)$ & $98.7(8.2)$ & 0.637 \\
\hline Waist-hip ratio & $0.880(0.039)$ & $0.873(0.095)$ & 0.870 \\
\hline \multicolumn{4}{|l|}{$\mathrm{BP}$ and pulse } \\
\hline \multicolumn{4}{|l|}{ Examination site } \\
\hline Systolic BP (mmHg) & $133.0(12.4)$ & $138.5(16.4)$ & 0.468 \\
\hline Diastolic BP (mmHg) & $84.3(10.9)$ & $89.9(8.1)$ & 0.281 \\
\hline Pulse (counts/min) & $66.3(4.4)$ & $69.9(3.4)$ & 0.106 \\
\hline \multicolumn{4}{|l|}{ Home in the morning } \\
\hline Systolic BP (mmHg) & $129.5(13.4)$ & $136.1(13.3)$ & 0.358 \\
\hline Diastolic BP (mmHg) & $84.0(7.2)$ & $89.4(11.1)$ & 0.279 \\
\hline Pulse (counts/min) & $64.1(5.4)$ & $68.5(6.0)$ & 0.158 \\
\hline \multicolumn{4}{|l|}{ Home at night } \\
\hline Systolic BP (mmHg) & $126.4(14.6)$ & $135.2(17.6)$ & 0.307 \\
\hline Diastolic BP (mmHg) & $74.4(12.8)$ & $86.1(12.6)$ & 0.099 \\
\hline Pulse (counts/min) & $71.9(12.0)$ & $75.3(11.4)$ & 0.592 \\
\hline
\end{tabular}

Figures denote average (standard deviation), unless otherwise specified

$B P$ blood pressure

a Unpaired $t$ test or Fisher's exact test

To compare the baseline characteristics between the polyphenol group and the placebo group, unpaired $t$ test was conducted for continuous variables, and Fisher's exact test for categorical variables. Two-way analysis of variance (ANOVA) was conducted for comparing the variation of BP, PCS and MCS between/among the groups during the study period. In each group, time-dependent repeated-measure analyses and Dunnett's test were performed [vs. data at baseline (week 0)]. In case Dunnett's test was unavailable, paired $t$ test was conducted instead. $p$-values (two-sided) less than 0.05 were considered statistically significant. All analyses were conducted using SAS software, version 9.1 (SAS Institute, Inc., Cary, NC, USA).

\section{Results}

Baseline characteristics

Shown are the baseline characteristics of study subjects in the polyphenol group and in the placebo group in Table 1 .

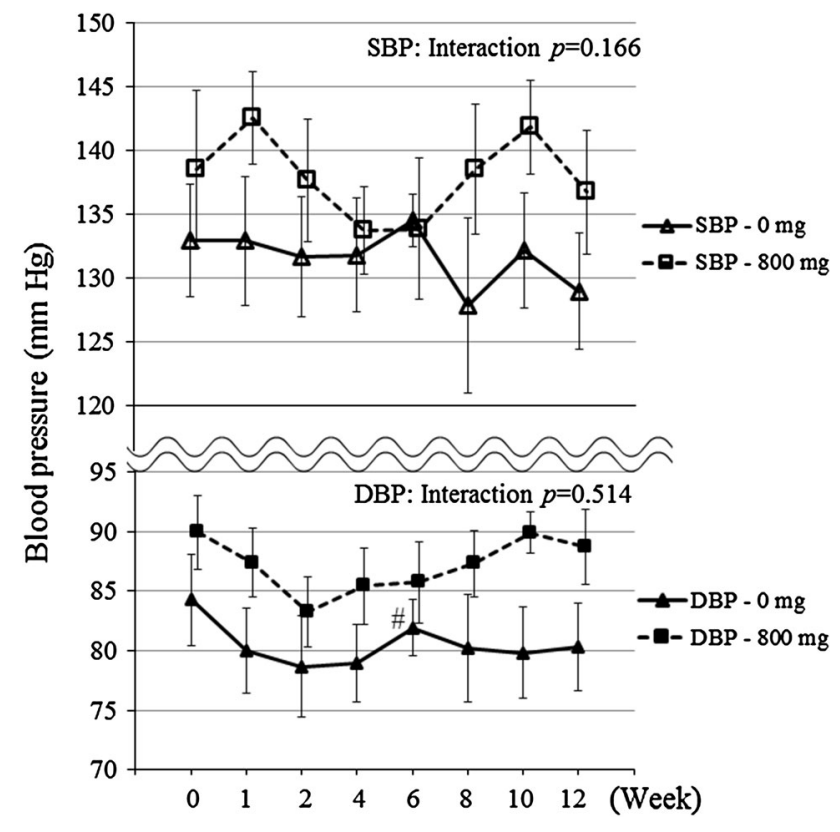

Fig. 2 Effect of Umezu polyphenols on blood pressure measured at the examination site. Time-dependent repeated-measure analyses and Dunnett's test were performed (vs. data in week 0). The interaction between intervention and time was also evaluated $\left.{ }^{\#} p<0.10\right)$. SBP systolic blood pressure, $D B P$ diastolic blood pressure

We did not observe significant differences between the two groups, which were partly due to the small number of the study subjects $(n=15)$.

Blood pressure at the examination site

Changes of BP in study subjects measured by research staff at the examination site are presented in Fig. 2. During the study period, the BP fluctuated in both the groups, resulting in a nonsignificant finding in SBP and DBP. The discrepancy of $\mathrm{BP}$ between the two groups remained during the study.

\section{Blood pressure at home}

Figure 3 shows changes in self-measured BP at home, (a) in the morning, and (b) at night. In the morning BP (Fig. 3a), SBP did not decrease in the polyphenol group, while DBP tended to decrease in both the groups, not reaching significance.

In the night BP (Fig. 3b), SBP and DBP fluctuated to produce some significant to quasi-significant values, although the interaction analysis did not show significant effects of UP on BP.

The discrepancy of BP between the two groups remained during the study. 


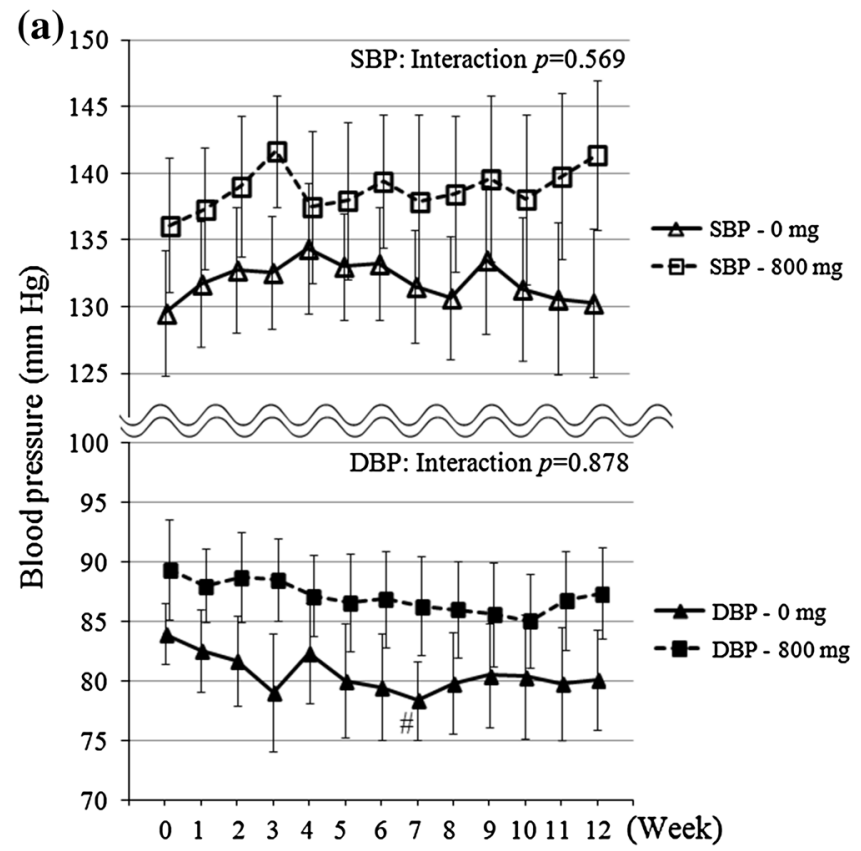

Fig. 3 Effect of Umezu polyphenols on blood pressure measured at home, $\mathbf{a}$ in the morning, and $\mathbf{b}$ at night. Time-dependent repeatedmeasure analyses and Dunnett's test were performed (vs. data in week

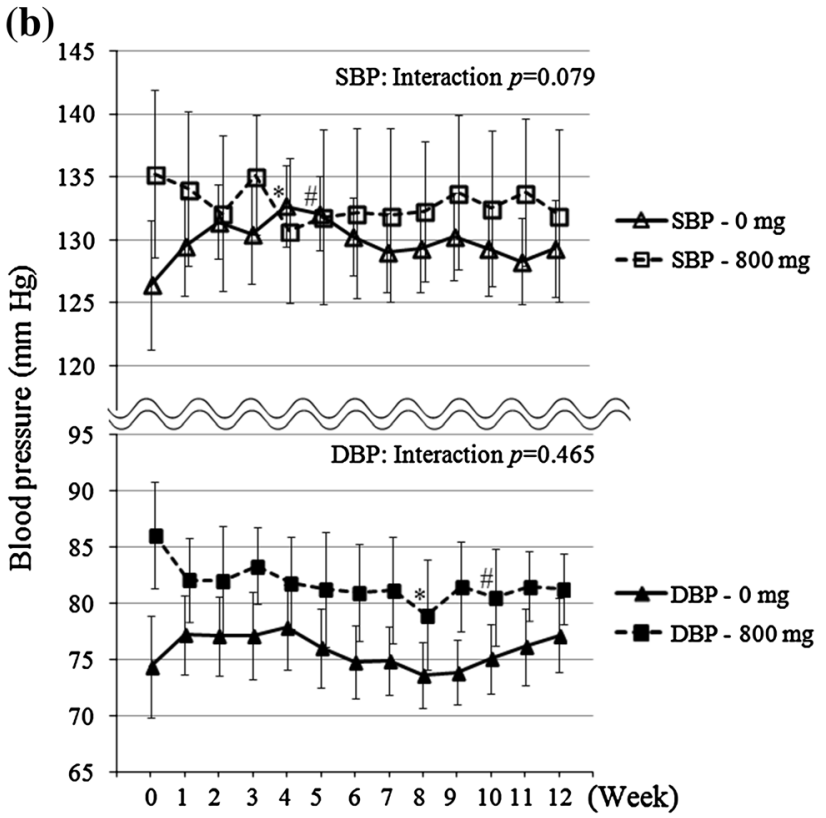

$0)$. The interaction between intervention and time was also evaluated $\left(* p<0.05 ;{ }^{*} p<0.10\right)$. SBP systolic blood pressure, DBP diastolic blood pressure

Table 2 Effects of Umezu polyphenols on physical and mental component summaries in SF-8

\begin{tabular}{|c|c|c|c|c|c|c|c|c|c|c|}
\hline & \multirow[t]{2}{*}{ UP (mg/day) } & \multicolumn{2}{|c|}{ Week 0} & \multicolumn{2}{|c|}{ Week 4} & \multicolumn{2}{|c|}{ Week 8} & \multicolumn{2}{|c|}{ Week 12} & \multirow[t]{2}{*}{ Interaction $p$} \\
\hline & & Mean & SD & Mean & SD & Mean & SD & Mean & SD & \\
\hline \multirow[t]{2}{*}{ PCS } & 0 & 52.19 & 5.13 & 52.08 & 3.78 & 51.28 & 6.13 & 49.07 & 5.29 & \multirow[t]{2}{*}{0.512} \\
\hline & 800 & 52.52 & 5.25 & 52.91 & 2.94 & 51.72 & 4.66 & 53.30 & 2.80 & \\
\hline \multirow[t]{2}{*}{ MCS } & 0 & 41.46 & 9.88 & 44.79 & 8.67 & 43.88 & 4.49 & 42.71 & 9.20 & \multirow[t]{2}{*}{0.231} \\
\hline & 800 & 49.75 & 3.80 & 52.42 & 2.17 & 52.79 & 2.87 & 50.22 & 2.88 & \\
\hline
\end{tabular}

Although time-dependent repeated-measure analyses and Dunnett's test were attempted (vs. data in week 0), analyses resulted in an error. Instead, summary scores in weeks 4,8 and 12 were separately compared with the data in week 0 with paired $t$ test, and none of them resulted in a significant outcome. Then, the interaction between intervention and time was evaluated

PCS physical component summary, MCS mental component summary, UP Umezu polyphenols

Safety and adherence

As for the safety of UP capsules, it was the winter season, when common cold was prevalent, and study subjects reported some signs and symptoms, mainly some respiratory and digestive ones. But these conditions were transient and recovered soon. And all the subjects competed the 12-week RCT. In SF-8, found were some nonsignificant improvements of the self-perceived physical condition in the polyphenol group, and some nonsignificant improvements of the self-perceived mental condition in both the groups (Table 2).

\section{Discussion}

In the current pilot study conducted in a double-blind randomized controlled trial (RCT), the beneficial effects of UP on hypertension were limited, probably due to the small number of study subjects. However, it showed the safety of UP capsules, even administered in a higher dose $(800 \mathrm{mg}$ daily) and for 12 weeks. Furthermore, the adherence of study subjects was good.

Wakayama Prefecture is higher in the mortality rate [20] and in the prevalence of cardiovascular diseases [21] compared with the average in Japan, which may be partly 
due to the high prevalence in residents aged 65 years or older. On the other hand, several surveys reveal that residents in Wakayama have the highest [22] and the second highest (next to Shizuoka) [23] household expenditures on Umeboshi (pickled plums) and Mandarin oranges, respectively, and plum [13, 14] as well as citrus intake [24] are negatively associated with cardiovascular disease. Japanese plum is consumed mainly in the form of Umeboshi, which contains a considerable amount of sodium, or in the form of Umeshu (a Japanese liqueur made from steeping Ume fruits (while still unripe and green) in alcohol and sugar). Sodium in Umeboshi may elevate BP, while daily salt intake in Wakayama is lower than the average in Japan [25], and Umeshu does not give plum active ingredients to nondrinkers. In other words, the way in which Japanese plum is consumed may not contribute to substantial reduction of cardiovascular disease. Therefore, some more efficient ways to ingest plum active ingredients are desired. UP extracts, free of sodium and alcohol, can be provided in soft drinks [15] and in capsules, and they could serve as a solution to provide beneficial effects of Japanese plum.

Our previous study in 2011 [15] was as short as 5 weeks, and the maximum dose of UP was as low as $200 \mathrm{mg}$ daily, which possibly led to the failure in showing significant and consistent findings regarding the BP-lowering effects of UP. To overcome these problems, a daily dose of $800 \mathrm{mg}$ and a study period of 12 weeks were adopted in this study. Due to the length of the study, and the exclusion of those with hypertension grade 2 or greater as well as those under medication, the number of study subjects was limited. Besides, we encouraged workers with high-normal BP or hypertension without medication to join the study and exclude those with medication, maximizing the effects of UP.

The study population from which the study subjects derived is the personnel of the institute the principal investigator (ST) belongs to, and is likely to understand and cooperate to the study in general. The previous 5-week study in 2011 was also conducted thanks to this population [15]. However, the present study was as long as 12 weeks. In general, the longer the period of a study is, the less likely they are to participate in it. In addition, not a few hypertensive candidates were reportedly excluded before the study enrollment due to taking antihypertensive agents, which suggests that the personnel of the institute is aware of their own health. These conditions resulted in a small number of subjects. However, given the scarcity of RCTs of fruit polyphenols in humans evaluating for 12 weeks or longer, this study will provide another piece of valuable evidence in the study of polyphenols.

The safety of UP in experimental animals is already shown in a series of studies including a 90-day subchronic study in rats [16]. And this is the first study where UP were administered for 12 weeks in humans. Liu et al. [26] showed in a meta-analysis that the number of studies on fruit juice polyphenol with a study period of 12 weeks or 3 months is limited [27-29], and that there is only one study evaluating the effects of fruit polyphenols on BP as long as 3 months [27], which showed no significant outcomes. But in the overall meta-analysis, fruit juice reduced DBP significantly by $2.07 \mathrm{mmHg} \quad(95 \%$ CI -3.75 , $-0.39 \mathrm{mmHg}$ ), but SBP insignificantly by $2.03 \mathrm{~mm} \mathrm{Hg}$ $(95 \% \mathrm{CI}-4.47,+0.41 \mathrm{mmHg}$ ). These results are partially consistent with findings of the present study. In another 12-week RCT among overweight Korean adults [30], a polyphenol extract from Ecklonia cava significantly contributed to lowering body fat and serum lipid parameters such as total and LDL cholesterols with dose dependence. It also had some BP-lowering effects, although its doseresponse effect was not significant. Interventional studies of a longer period evaluating the effects of polyphenols among humans are very limited. In a 16-week study among patients under antihypertensive medication, BP reduction was observed in the extra-virgin olive oil group compared with the sunflower oil group. Enhanced nitric oxide levels stimulated by polyphenols might contribute to this beneficial effect [31].

As for SF-8, the physical condition improved in the polyphenol group and the mental condition improved in both the groups. In our previous study, the physical condition improved only in the polyphenol group and the mental condition improved only in the placebo group [15]. Given some placebo effects in the placebo group, these findings support some physically positive effects in UP. The biology of this improvement is yet to be clarified.

Several reasons may explain why the effects of UP against BP were limited. First, involvement of type 2 error should be taken into account. This RCT was a preliminary test, and it was conducted in a small number of subjects. This might have reduced the power to detect BP-lowering effects of UP. Second, some psychological factors might be involved in the decrease of BP in the placebo group, although it is unclear why this occurred. In our previous study [15], the MCS score significantly improved in the placebo group, but it did not in the UP group, which resulted in the diminished differences between UP and placebo groups.

Some study limitations should be mentioned. First, information on the subjects' age and sex was obtained during the recruitment of study participants and before the random allocation, but their BP was measured only after the random allocation and the beginning of the study, due to some logistic reasons. Therefore, baseline BP could not be utilized as the allocation variable. Second, it should be considered that study subjects ignored some "outliers" in BP since they were medical professionals; they might 
reject some BP values that they subjectively considered extremely high or low as incorrect rather than recorded them "as is", and then measured BP repeatedly until they got values that they could regard as correct. These circumstances could throw some effects on the discrepancy in BP.

To overcome the above-mentioned limitations, we conducted a 14-week community-based RCT in 2013, where we randomly allocated a total of 72 study subjects into two arms, taking into account of their baseline BP as well as sex and age. Furthermore, to ensure objectivity in home BP self-measurement, we introduced a personal BP monitor which allowed home measurement data to be automatically transmitted to servers over $3 \mathrm{G}$ networks. We would like to report the results later.

In conclusion, the ability of UP to decrease BP is no more remarkable than that of a placebo. However, UP were shown safe and high in adherence, even administered in a higher dose (800 mg daily) and for a longer period (12 weeks).

Acknowledgments This work was supported by Japan Society for the Promotion of Science (JSPS KAKENHI Grant Number 24790590). In the process of the manuscript preparation, some cordial advices were given from Dr. Naomi Kitano at the Department of Public Health, School of Medicine, Wakayama Medical University.

Conflict of interest The authors declare that they have no conflict of interest.

\section{References}

1. Ueshima H. Explanation for the Japanese paradox: Prevention of increase in coronary heart disease and reduction in stroke. J Atheroscler Tromb. 2007;14:278-86.

2. Japanese Society of Hypertension. Guidelines for the Management of Hypertension. Tokyo: Life Science Publishing Co., Ltd.; 2009 (In Japanese).

3. Ness AR, Powles JW. Fruit and vegetables, and cardiovascular disease: a review. Int J Epidemiol. 1997;26:1-13.

4. Ness A, Egger M, Powles J. Fruit and vegetables and ischemic heart disease: systematic review or misleading meta-analysis? Eur J Clin Nutr. 1999;53:900-4.

5. Sasazuki S. Fukuoka Heart Study Group. Case-control study of nonfatal myocardial infarction in relation to selected foods in Japanese men and women. Jpn Circ J. 2001;65:200-6.

6. Schiffrin EL. Antioxidants in hypertension and cardiovascular disease. Mol Interv. 2010;10:354-62.

7. Rapola JM, Virtamo J, Ripatti S, Huttunen JK, Albanes D, Taylor PR, et al. Randomised trial of alpha-tocopherol and betacarotene supplements on incidence of major coronary events in men with previous myocardial infarction. Lancet. 1997;349:1715-20.

8. Curin Y, Andriantsitohaina R. Polyphenols as potential therapeutical agents against cardiovascular diseases. Pharmacol Rep. 2005;57:97-107.

9. Schini-Kerth VB, Etienne-Selloum N, Chataigneau T, Auger C. Vascular protection by natural product-derived polyphenols: in vitro and in vivo evidence. Planta Med. 2011;77:1161-7.
10. Galleano M, Pechanova O, Fraga CG. Hypertension, nitric oxide, oxidants, and dietary plant polyphenols. Curr Pharm Biotechnol. 2010;11:837-48.

11. Michalska M, Gluba A, Mikhailidis DP, Nowak P, BieleckaDabrowa A, Rysz J, et al. The role of polyphenols in cardiovascular disease. Med Sci Monit. 2010;16:110-9.

12. Ahmed T, Sadia H, Batool S, Janjua A, Shuja F. Use of prunes as a control of hypertension. J Ayub Med Coll Abbottabad. 2010;22:28-31.

13. Enomoto S, Yanaoka K, Utsunomiya H, Niwa T, Inada K, Deguchi $\mathrm{H}$, et al. Inhibitory effects of Japanese apricot (Prunus mume Siebold et Zucc.; Ume) on Helicobacter pylori-related chronic gastritis. Eur J Clin Nutr. 2010;64:714-9.

14. Utsunomiya H, Takekoshi S, Gato N, Utatsu H, Motley ED, Eguchi K, et al. Fruit-juice concentrate of Asian plum inhibits growth signals of vascular smooth muscle cells induced by angiotensin II. Life Sci. 2002;72:659-67.

15. Takemura S, Yoshimasu K, Mure K, Fukumoto J, Nishio N, Kitano N, et al. Are Umezu polyphenols in the Japanese plum (Prunus Mume) protective against mild hypertension and oxidation? Evidence from a double-blind randomized placebo-controlled trial. Open J Prev Med. 2013;3:561-9.

16. Standards and Evaluation Division, Department of Food Safety, Pharmaceutical and Food Safety Bureau, Ministry of Health, Labor and Welfare, Government of Japan. Important Notice about Prepartion of Attached Documents in Applications for Reviews of Foods for Specified Health Uses (FOSHU). Notification, Shokuanshinhatsu 0201002. Issued on February 1, 2005. Retrieved on July 28, 2014, from http://www.caa.go.jp/foods/pdf/ syokuhin92.pdf (In Japanese).

17. Shiga Y, Tsuchida T, Hara Y, Kishida K, Maeda M, Miyashita K, et al. Safety studies of Umezu Polyphenol Extract. Mem Fac Biol Oriented Sci Technol Kinki Univ. 2011;28:31-40 (In Japanese).

18. Mitani T, Horinishi A, Kishida K, Kawabata T, Yano F, Mimura $\mathrm{H}$, et al. Phenolics profile of mume, Japanese apricot (Prunus mume Sieb. et Zucc.) fruit. Biosci Biotechnol Biochem. 2013;77:1623-7.

19. Fukuhara S, Suzukamo Y. Manual of the SF-8 Japanese Version. Kyoto: Institute for Health Outcomes \& Process Evaluation Research; 2004 (in Japanese).

20. Statistics and Information Department, Minister's Secretariat, Ministry of Health, Labour and Welfare, Government of Japan. Summaries of Monthly Reports of Vital Statistics (Provisional Estimates) in 2013. 2014. Retrieved on July 28, 2014, from http:// www.mhlw.go.jp/toukei/saikin/hw/jinkou/geppo/nengai13/index. html (In Japanese).

21. Wakayama Prefecture Government. Health Planning in Wakayama Prefecture. 2013. Retrieved on July 28, 2014, from http:// www.pref.wakayama.lg.jp/prefg/050100/iryokeikaku/keikaku. html (In Japanese).

22. Statistics Bureau, Ministry of Internal Affairs and Communications, Government of Japan. Household Expenditure on Umeboshi (pickled plums): Results from Family Income and Expenditure Survey (Two-or-more-person Households). 2010. Retrieved on July 28, 2014, from http://www.stat.go.jp/data/ kakei/tsushin/pdf/22_6.pdf (In Japanese).

23. Shizuoka Prefecture Government. Shizuoka is the highest in the household expenditure on and the household purchased amount of Mandarin orange. Updated on May 5, 2014. Retrieved on July 28, 2014, from http://www.pref.shizuoka.jp/j-no1/mikan_sisyutu. html (In Japanese).

24. Takachi R, Inoue M, Ishihara J, Kurahashi N, Iwasaki M, Sasazuki S, Iso H, Tsubono Y, Tsugane S. JPHC Study Group. Fruit and vegetable intake and risk of total cancer and cardiovascular disease: Japan Public Health Center-Based Prospective Study. Am J Epidemiol. 2008;167:59-70. 
25. The Ministry of Health, Labor and Welfare, Government of Japan. The National Health and Nutrition Survey in Japan, 2012. 2014. Retrieved on July 28, 2014, from http://www.mhlw.go.jp/ bunya/kenkou/eiyou/h24-houkoku.html (In Japanese).

26. Liu K, Xing A, Chen K, Wang B, Zhou R, Chen S, et al. Effect of fruit juice on cholesterol and blood pressure in adults: a metaanalysis of 19 randomized controlled trials. 2013. PLoS One. 2013;8:e61420.

27. Sumner MD, Elliott-Eller M, Weidner G, Daubenmier JJ, Chew $\mathrm{MH}$, Marlin R, et al. Effects of pomegranate juice consumption on myocardial perfusion in patients with coronary heart disease. Am J Cardiol. 2005;96:810-4.

28. Hollis JH, Houchins JA, Blumberg JB, Mattes RD. Effects of concord grape juice on appetite, diet, body weight, lipid profile, and antioxidant status of adults. J Am Coll Nutr. 2009;28:574-82.
29. Aptekmann NP, Cesar TB. Orange juice improved lipid profile and blood lactate of overweight middle-aged women subjected to aerobic training. Maturitas. 2010;67:343-7.

30. Shin HC, Kim SH, Park Y, Lee BH, Hwang HJ. Effects of 12-week oral supplementation of Ecklonia cava polyphenols on anthropometric and blood lipid parameters in overweight Korean individuals: a double-blind randomized clinical trial. Phytother Res. 2012;26:363-8.

31. Ferrara LA, Raimondi AS, d'Episcopo L, Guida L. Dello Russo A, Marotta T. Olive oil and reduced need for antihypertensive medications. Arch Intern Med. 2000;160:837-42. 